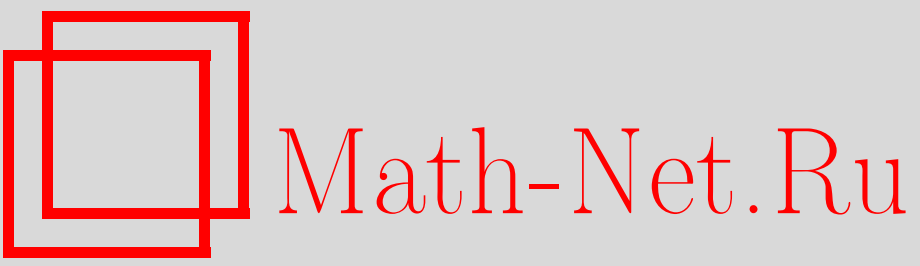

K. В. Степаньянц, О невозможности исключения калибровочных степеней свободы из точного суперпотенциала при $N_{c}>N_{f}, T M \Phi, 2000$, том 122 , номер 3, 435-443

DOI: https://doi.org/10.4213/tmf578

Использование Общероссийского математического портала Math-Net.Ru подразумевает, что вы прочитали и согласны с пользовательским соглашением

http: //www.mathnet.ru/rus/agreement

Параметры загрузки:

IP : 18.208 .226 .222

26 апреля 2023 г., 16:24:55 
ТЕОРЕТИЧЕСКАЯ

И МАТЕМАТИЧЕСКАЯ

ФИЗИКА

Том 122, № 3

март, 2000

(C) 2000 г.

К. В. Степаньянц*

\section{О НЕВОЗМОЖНОСТИ ИСКЛЮЧЕНИЯ КАЛИБРОВОЧНЫХ СТЕПЕНЕЙ СВОБОДЫ ИЗ ТОЧНОГО СУПЕРПОТЕНЦИАЛА ПРИ $N_{c}>N_{f}$}

Изучается непертурбативное обобщение эффективного лагранжиана ВенецианоЯнкиеловича. Показано, что переход от него к суперпотенциалу Аффлека-Дайна-Зайберга исключением конденсата глюино на уравнениях движения невозможен при $N_{c}>N_{f}$.

\section{1. ВВЕДЕНИЕ}

Понимание непертурбативной динамики теории поля крайне важно для объяснения целого ряда явлений - конфайнмента кварков, проблемы калибровочной иерархии, нарушения суперсимметрии. В настояшее время в данной области достигнут определенный прогресс. Прежде всего он связан с результатами работы [1], в которой впервые была вычислена сумма ряда инстантонных поправок для наиболее простой модели - $N=2$ суперсимметричной теории Янга-Миллса с калибровочной группой $S U(2)$. Как было показано в работах [2-4], асимптотика данного результата действительно правильно воспроизводит одно- и двухинстантонную поправки.

Однако в реалистичных моделях реализуется не $N=2$, а $N=1$ суперсимметрия [5], поэтому наибольший интерес представляет именно этот случай. Впервые непертурбативные эффекты в $N=1$ суперсимметричных теориях были рассмотрены в работе [6], идеи которой получили интенсивное развитие в последнее время (см., например, [7]). Однако соответствующие результаты не согласуются, во-первых, с прямыми инстантонными вычислениями и, во-вторых, с формулой, связывающей эффективное действие и квантовые аномалии. Решение данных проблем было предложено в работах [8, 9]. Было показано, что эффективное действие должно обязательно зависеть от калибровочного поля, поскольку тогда удается не только достичь согласия с законом преобразования меры интегрирования по коллективным координатам, но и воспроизвести аномалии киральных симметрий теории. Результат фактически представляет собой непертурбативное обобщение эффективного лагранжиана Венециано-Янкиеловича [10].

\footnotetext{
* Московский государственный университет, Москва, Россия. E-mail: stepan@theor.phys.msu.su
} 
Однако эффективный лагранжиан Венециано-Янкиеловича зависит не от исходных полей, а от некоторого связанного с ними нового калибровочно-инвариантного скалярного суперполя $S$, так называемого конденсата глюино, который обычно рассматривается как новая степень свободы. Известно [11, 8], что при его исключении на уравнениях движения получаются точные результаты Зайберга при $N_{f} \geqslant N_{c}$ или Аффлека-Дайна-Зайберга при $N_{f}<N_{c}$. Но при этом само это исключение должно быть возможно. Это действительно так, пока задача рассматривается в рамках теории возмущений, однако эффективный лагранжиан Венециано-Янкиеловича не применим в непертурбативной области. В частности, анализ возможности исключения конденсата глюино должен проводиться на основе точного выражения для эффективного действия. Такой анализ проведен в настоящей работе.

Статья организована следующим образом. В разделе 2 мы напоминаем основные сведения о непертурбативном обобщении эффективного лагранжиана Венециано-Янкиеловича. В разделе 3 исследуется возможность исключения конденсата глюино на уравнениях движения. В разделе 4 кратко обсуждаются полученные результаты.

\section{2. НЕПЕРТУРБАТИВНОЕ ОБОБЩЕНИЕ ЭФФЕКТИВНОГО ЛАГРАНЖИАНА ВЕНЕЦИАНО-ЯНКИЕЛОВИЧА}

Мы будем рассматривать безмассовую $N=1$ суперсимметричную теорию ЯнгаМиллса с калибровочной группой $S U\left(N_{c}\right)$ и $N_{f}$ мультиплетами материи, которая в суперпространстве описывается действием

$$
S=\frac{1}{16 \pi} \operatorname{tr} \operatorname{Im}\left(\tau \int d^{4} x d^{2} \theta W^{2}\right)+\frac{1}{4} \int d^{4} x d^{4} \theta \sum_{A=1}^{N_{f}}\left(\phi_{A}^{+} e^{-2 V} \phi^{A}+\tilde{\phi}^{+A} e^{2 V} \tilde{\phi}_{A}\right)
$$

(суперполя материи $\phi$ и $\tilde{\phi}$ принадлежат фундаментальному и антифундаментальному представлениям калибровочной группы $S U\left(N_{c}\right)$ ). Здесь были использованы следующие обозначения:

$$
\begin{aligned}
\phi(y, \theta)= & \varphi(y)+\sqrt{2} \bar{\theta}\left(1+\gamma_{5}\right) \psi_{1}(y)+\frac{1}{2} \bar{\theta}_{1}\left(1+\gamma_{5}\right) \theta f(y), \\
V(x, \theta)= & -\frac{i}{2} \bar{\theta} \gamma^{\mu} \gamma_{5} \theta A_{\mu}(x)+i \sqrt{2}(\bar{\theta} \theta)\left(\bar{\theta} \gamma_{5} \psi_{2}(x)\right)+\frac{i}{4}(\bar{\theta} \theta)^{2} D \\
W(y, \theta)= & \frac{1}{2}\left(1+\gamma_{5}\right)\left(i \sqrt{2} \psi_{2}(y)+i \theta D(y)+\frac{1}{2} \Sigma_{\mu \nu} \theta F_{\mu \nu}(y)+\right. \\
& \left.+\frac{1}{\sqrt{2}} \bar{\theta}\left(1+\gamma_{5}\right) \theta \gamma^{\mu} D_{\mu} \psi_{2}(y)\right) \\
\tau= & \frac{\theta}{2 \pi}+\frac{4 \pi i}{e^{2}}, \quad y^{\mu}=x^{\mu}+\frac{i}{2} \bar{\theta} \gamma^{\mu} \gamma_{5} \theta .
\end{aligned}
$$

Эффективный лагранжиан данной модели может быть представлен в виде суммы [10]:

$$
L_{\mathrm{eff}}=L_{\mathrm{k}}+L_{\mathrm{a}}
$$


где

$$
L_{\mathrm{k}}=\int d^{4} \theta K\left(S, S^{*}, \Phi, \Phi^{*}\right), \quad L_{\mathrm{a}}=\operatorname{Re} \int d^{2} \theta w(S, \Phi)
$$

и $S \equiv \operatorname{tr} W^{2}$ - конденсат глюино, который в силу (2) включает в себя калибровочное поле и, следовательно, может рассматриваться как калибровочная степень свободы. Зависимость от $S$ (которая не исследовалась в [6]) необходима для достижения согласия с законом преобразования инстантонной меры и воспроизведения аномалий киральных симметрий.

В эффективном лагранжиане $(3) L_{\mathrm{k}}$ обозначает кинетический член, которьй не дает вклада в аномалию, а $L_{\mathrm{a}}$ - голоморфная часть суперпотенциала. В теории возмущений к $L_{\mathrm{k}}$ существуют нетривиальные поправки во всех петлях, и данное слагаемое, конечно, не может быть точно вычислено даже на пертурбативном уровне. Однако с использованием аргументов, основанных на исследовании квантовых аномалий, оказывается возможным определить вид $L_{\mathrm{a}}$. Это может быть сделано как в рамках теории возмущений $[10]$, так и точно (с учетом ряда инстантонных поправок) $[9]^{1)}$.

Исследование закона преобразования меры интегрирования под действием аномальной симметрии $U(1)_{x}$

$$
\begin{aligned}
W(\theta) & \rightarrow e^{i \alpha} W\left(e^{-i \alpha \gamma_{5}} \theta\right), \\
\phi(\theta) & \rightarrow e^{i x \alpha} \phi\left(e^{-i \alpha \gamma_{5}} \theta\right), \\
\tilde{\phi}(\theta) & \rightarrow e^{i x \alpha} \tilde{\phi}\left(e^{-i \alpha \gamma_{5}} \theta\right)
\end{aligned}
$$

( $x$ - произвольная постоянная) позволяет заключить [9], что

$$
L_{\mathrm{a}}=\frac{1}{32 \pi^{2}} \operatorname{Im} \operatorname{tr} \int d^{2} \theta W^{2} g(z)
$$

где (с учетом требования положительной определенности евклидова действия)

$$
g(z)=-i \ln z+\sum_{k=0}^{\infty} c_{n} z^{n}, \quad \operatorname{Im} g(z) \geqslant 0, \quad \operatorname{Re} c_{n}=0
$$

а параметр $z$ является функцией “мезонов" $M$ и “барионов” $B$, определяемых как

$$
\begin{aligned}
M_{A}^{B} & =\tilde{\phi}_{A a} \phi^{B a}, \\
B_{A_{N_{c}+1} A_{N_{c}+2} \ldots A_{N_{f}}} & =\frac{1}{N_{c} !} \varepsilon_{A_{1} A_{2} \ldots A_{N_{f}}} \varepsilon^{a_{1} a_{2} \ldots a_{N_{c}} \phi^{A_{1} a_{1}} \phi^{A_{2} a_{2}} \ldots \phi^{A_{N_{c}} a_{N_{c}}},} \\
\widetilde{B}^{A_{N_{c}+1} A_{N_{c}+2} \ldots A_{N_{f}}} & =\frac{1}{N_{c} !} \varepsilon^{A_{1} A_{2} \ldots A_{N_{f}}} \varepsilon^{a_{1} a_{2} \ldots a_{N_{c}}} \tilde{\phi}_{A_{1} a_{1}} \tilde{\phi}_{A_{2} a_{2}} \ldots \tilde{\phi}_{A_{N_{c}} a_{N_{c}}},
\end{aligned}
$$

\footnotetext{
1) Из-за существования инстантонных вкладов точные аномалии существенно отличаются от пертурбативных (см. [12]).
} 
а также конденсата глюино $S=W^{2}$. Первое слагаемое и нулевой член суммы $c_{0}$ в выражении (7) представляют собой пертурбативный результат, а члены, содержашие степени $z,-$ инстантонные поправки. Более детальное обсуждение данных вопросов дается в работе [8], где были получены следующие выражения:

$$
\begin{gathered}
z=\frac{\Lambda^{3 N_{c}-N_{f}}}{\operatorname{det} M S^{N_{c}-N_{f}}}, \quad N_{c}>N_{f}, \\
z=\frac{\Lambda^{3 N_{c}-N_{f}} S^{N_{f}-N_{c}}}{\operatorname{det} M-\left(\widetilde{B}^{A_{1} A_{2} \ldots A_{N_{f}-N_{c}}} M_{A_{1}} B_{1} M_{A_{2}} B_{2} \ldots M_{A_{N_{f}-N_{c}}}^{\left.B_{N_{f}-N_{c}} B_{B_{1} B_{2} \ldots B_{N_{f}-N_{c}}}\right)}\right.}, \\
N_{c} \leqslant N_{f} .
\end{gathered}
$$

Из условий (7) однозначно следует [13], что

$$
g(z)=2 \pi \tau\left(z^{-\frac{1}{4}}\right)
$$

где $\tau(a)$ совпадает с соответствуюшей функцией в решении Зайберга-Виттена, определяемой как

$$
\tau(a)=\left.\frac{d a_{D}(u)}{d a}\right|_{u=u(a)}
$$

а функции $a(u)$ и $a_{D}(u)$ суть

$$
a(u)=\frac{\sqrt{2}}{\pi} \int_{-1}^{1} d x \frac{\sqrt{x-u}}{\sqrt{x^{2}-1}}, \quad a_{D}(u)=\frac{\sqrt{2}}{\pi} \int_{1}^{u} d x \frac{\sqrt{x-u}}{\sqrt{x^{2}-1}} .
$$

Доказательство этого утверждения приведено в [13]. В качестве исходного используется уравнение

$$
u=\pi i\left(F(a)-\frac{a}{2} \frac{\partial F(a)}{\partial a}\right),
$$

где $a=z^{-1 / 4}$, а функция $F(a)$ определяется с помошњю соотношения $g(a) \equiv 2 \pi F^{\prime \prime}(a)$. При этом $u$ должно удовлетворять следуюшим свойствам:

$$
u(g)^{*}=u\left(-g^{*}\right), \quad u(g-2 \pi n)=(-1)^{n} u(g) .
$$

С физической точки зрения уравнение (13) представляет собой аномальное тождество Уорда, получающееся при дифференцировании эффективного лагранжиана Зайберга-Виттена по параметру $R$-симметрии [14]. Аналогичное уравнение может быть выведено и в модели $(1)$ на основе исследования аномалии $U(1)_{x}$-симметрии $[9]$. Поэтому совпадение формы эффективного действия для совершенно различных моделей фактически является следствием того, что выражения для квантовых аномалий сходны по форме.

С точки зрения математики уравнение (13) является следствием разложения (7), поскольку из него мы находим, что

$$
F(a)=a^{2}\left(\frac{i}{\pi} \ln a+\sum_{k=0}^{\infty} F_{k} a^{-4 k}\right), \quad \operatorname{Re} F_{k}=0
$$


и, следовательно,

$$
u(a)=a^{2}\left(\frac{1}{2}+\sum_{k=1}^{\infty} 2 \pi i k F_{k} a^{-4 k}\right) .
$$

Первое из условий (14) следует из того, что коэффициенты $F_{k}$ являются чисто мнимыми, откуда $g(a)^{*}=-g\left(a^{*}\right)$. Поэтому $a(g)^{*}=a\left(-g^{*}\right)$, и требуемое условие получается подстановкой данного равенства в (16).

Для того чтобы вывести второе из условий (14), необходимо совершить преобразования

$$
a \rightarrow e^{\frac{i \pi n}{2}} a
$$

которые приводят к

$$
F(a) \rightarrow e^{i \pi n}\left(F(a)-\frac{n}{2} a^{2}\right), \quad g \rightarrow g-2 \pi n, \quad u \rightarrow(-1)^{n} u
$$

Таким образом, разложение (7) действительно эквивалентно исходным предположениям работы [13], и, следовательно, для голоморфной части эффективного лагранжиана окончательно получаем

$$
L_{\mathrm{a}}=\frac{1}{16 \pi} \operatorname{Im} \int d^{2} \theta S \tau\left(z^{-\frac{1}{4}}\right)
$$

В пределе $z \rightarrow 0$, соответствуюшем применимости теории возмушений, решение (19) переходит в эффективный лагранжиан Венециано-Янкиеловича [10]

$$
L_{\mathrm{VY}}=\frac{1}{32 \pi^{2}} \operatorname{Re} \int d^{2} \theta S(\ln z+\text { const })
$$

поскольку

$$
\tau(a) \rightarrow \frac{2 i}{\pi} \ln (2 a), \quad a \rightarrow \infty
$$

(Однако вне рамок теории возмушений эффективный лагранжиан Венециано-Янкиеловича не применим.)

Зависимость решения (19) от конденсата глюино $S$ (который в силу (2) включает в себя калибровочное поле) позволяет добиться выполнения условия связи между аномалиями (с учетом инстантонных поправок) и эффективным лагранжианом

$$
\left\langle\partial_{\mu} J^{\mu}\right\rangle=-\frac{\partial L_{\mathrm{a}}}{\partial \alpha}
$$

для произвольного значения параметра $x$ в преобразованиях (5). Выражения для $L_{\mathrm{a}}$, которые были предложены в работах [6,7], удовлетворяют условию (22) только при $x=\left(N_{f}-N_{c}\right) / N_{f}$ (в этом случае аномалия соответствуюшего тока равна нулю).

В рамках предложенного Зайбергом подхода "мезоны" и "барионы", исходно строяшиеся калибровочно-инвариантным образом из материальных полей теории, являются 
(при $N_{f} \geqslant N_{c}$ ) некоторыми новыми квантовыми полями так же, как и конденсат глюино, который всегда считается некоторым новым полем. Стандартно предполагается [11], что $S$ исключается с помошњю уравнений движения

$$
\frac{\partial w}{\partial S}=0
$$

Поскольку суперпотенциал $w$ удовлетворяет уравнению [9]

$$
2 w-2 \frac{\partial w}{\partial S} S-\frac{N_{f}-N_{c}}{N_{f}} \frac{\partial w}{\partial v}=0,
$$

то после наложения условия (23) получим уравнение, которое использовалось в работах $[6,7]$. Следовательно, если конденсат глюино может быть выражен из (23), то его подстановка в $w$ автоматически приводит к суперпотенциалу Аффлека-Дайна-Зайберга. Таким образом, решение (19) представляет собой синтез точных результатов Зайберга и эффективного лагранжиана Венециано-Янкиеловича. Однако оно применимо вне рамок теории возмущений, согласуется с законом преобразования меры интегрирования по коллективным координатам и воспроизводит квантовые аномалии.

\section{3. АНАЛИЗ ВОЗМОЖНОСТИ ИСКЛЮЧЕНИЯ КОНДЕНСАТА ГЛЮИНО НА УРАВНЕНИЯХ ДВИЖЕНИЯ}

Условие экстремума суперпотенциала (23) может быть переписано как $\left(a=z^{-1 / 4}\right)$

$$
\frac{d \ln a}{d \ln \tau}=\frac{1}{4}\left(N_{f}-N_{c}\right) .
$$

Используя пертурбативньй результат (21), в рамках теории возмущений мы немедленно получаем, что

$$
\ln (2 a)=\frac{1}{4}\left(N_{f}-N_{c}\right)
$$

Уравнение (26), конечно, имеет решения при любых значениях $N_{f}$ и $N_{c}$, поэтому в рамках теории возмушений исключение конденсата глюино на уравнениях движения является возможным, как это и предполагалось в [11]. Однако вне рамок теории возмушений ситуация кардинально меняется.

Для проведения соответствующего исследования нам потребуется представление решения Зайберга-Виттена через эллиптические функции [15]

$$
a(u)=\frac{4}{\pi k} E(k), \quad a_{D}(u)=\frac{4}{i \pi k}\left(E^{\prime}(k)-K^{\prime}(k)\right),
$$

где $k^{2}=2 /(1+u)$, функции $E, K, E^{\prime}$ и $K^{\prime}$ определяются как

$$
\begin{aligned}
E(k) & =\frac{\pi}{2} F\left(-\frac{1}{2}, \frac{1}{2}, 1, k^{2}\right)=\int_{0}^{1} d t \frac{\sqrt{1-k^{2} t^{2}}}{\sqrt{1-t^{2}}} \\
K(k) & =\frac{\pi}{2} F\left(\frac{1}{2}, \frac{1}{2}, 1, k^{2}\right)=\int_{0}^{1} d t \frac{1}{\sqrt{\left(1-k^{2} t^{2}\right)\left(1-t^{2}\right)}}, \\
E^{\prime}(k) & =E\left(\sqrt{1-k^{2}}\right), \quad K^{\prime}(k)=K\left(\sqrt{1-k^{2}}\right),
\end{aligned}
$$


а $F$ - гипергеометрическая функция. Функцию $\tau$ при этом можно записать в виде

$$
\tau=\frac{i K^{\prime}}{K}
$$

Преимушество такой формы записи заключается в том, что все данные специальные функции “зашиты” в системах аналитических вычислений. При выполнении настоящей работы использовалась система аналитических вычислений MAPPLE. C ее помощью был построен график функции

$$
\frac{d \ln a}{d \ln \tau},
$$

приведенный на рисунке (кривая 1), где для сравнения показан соответствуюший пертурбативный результат (кривая 2).

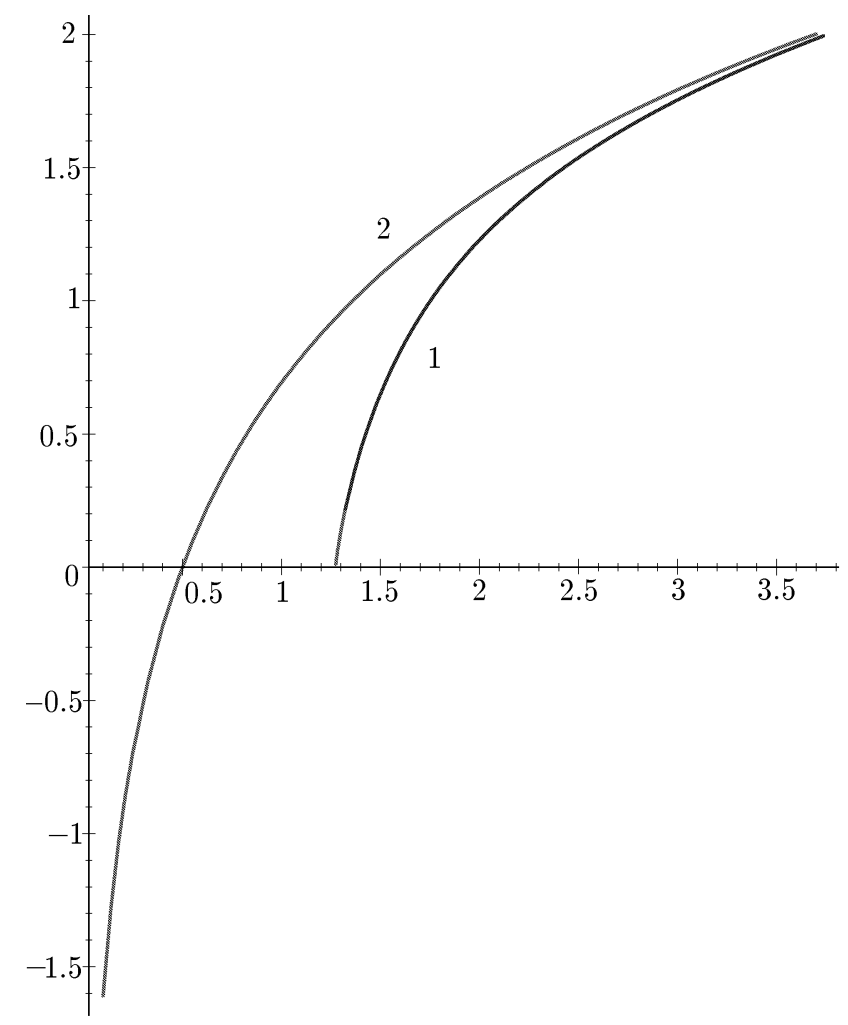

Графики левой части уравнения (25) как функции переменной $a$. Кривая 1 соответствует точному результату, а кривая 2 - результату, полученному в рамках теории возмушений.

Как и должно быть, при $a \rightarrow \infty$ результат теории возмущений практически совпадает с точным результатом, однако при малых значениях $a$ возникают принципиальные расхождения. Эллиптические функции вешественны только при $k^{2}=2 /(1+u)<1$. В 
области, где теория возмушений не применима $\left(k^{2} \rightarrow 0\right)$, функция (30) также всегда положительна и обрашается в нуль при $k^{2}=0$, что соответствует $a=4 / \pi$. Следовательно, область значений функции (30) для точного результата есть $[0, \infty)$, и решение уравнения (25) существует только при неотрицательной правой части, т.е. при $N_{c} \leqslant N_{f}$.

Отдельного упоминания заслуживает случай $N_{c}=N_{f}$. В этом случае классическое условие связи $\operatorname{det} M-\widetilde{B} B=0$ нарушается квантовыми поправками [7] как

$$
\operatorname{det} M-\widetilde{B} B=\operatorname{const} \Lambda^{2 N_{f}} .
$$

В рамках подхода, предложенного в работе [8], последнее условие очевидно выполняется, поскольку в данном случае

$$
L_{\mathrm{a}}=\frac{1}{16 \pi} \operatorname{Im} \int d^{2} \theta S \tau\left(\left(\frac{\Lambda^{2 N_{f}}}{\operatorname{det} M-\widetilde{B} B}\right)^{-\frac{1}{4}}\right),
$$

и, следовательно, конденсат глюино является просто множителем Лагранжа. При варьировании по нему получим уравнение $\tau(a)=0$, которое, как несложно проверить, имеет решение

$$
a=\left(\frac{\Lambda^{2 N_{f}}}{\operatorname{det} M-\widetilde{B} B}\right)^{-\frac{1}{4}}=\frac{4}{\pi} .
$$

Поэтому окончательно в случае $N_{c}=N_{f}$ получаем

$$
\operatorname{det} M-\widetilde{B} B=\left(\frac{4}{\pi}\right)^{4} \Lambda^{2 N_{f}}
$$

\section{4. ЗАКЛЮЧЕНИЕ}

Итак, в данной работе показано, что форма точного суперпотенциала такова, что при $N_{c}>N_{f}$ исключение конденсата глюино на уравнениях движения невозможно - в этом случае экстремум просто отсутствует. Следовательно, вопрос об эквивалентности точных результатов при наличии конденсата и без него отнюдь не столь тривиален, как это предполагалось в [11].

Возможность такой ситуации уже рассматривалась в работе [8] на основе некоторых качественных рассуждений. Дело в том, что при $N_{c}>N_{f}$ калибровочная группа не является полностью нарушенной и в теории присутствуют безмассовые калибровочные поля. С другой стороны, исключение конденсата на уравнениях движения предполагает замену исходных переменных теории на некоторые новые калибровочно-инвариантные величины. Конечно, это, по меньшей мере, достаточно неестественно при наличии ненарушенной калибровочной группы. Следовательно, при $N_{c}>N_{f}$, по-видимому, невозможно игнорировать зависимость эффективного действия от калибровочных степеней свободы, что делается при выводе суперпотенциала Аффлека-Дайна-Зайберга. Отсюда, в частности, и возникают проблемы с согласием точных результатов и инстантонных вычислений.

Таким образом, необходимо сделать вывод о неэквивалентности точного результата (19) и эффективного суперпотенциала Афффлека-Дайна-Зайберга при $N_{c}>N_{f}$. При $N_{c} \leqslant N_{f}$ (полностью нарушенная калибровочная группа) согласие по-прежнему существует. 
Благодарности. Я хотел бы выразить свою глубокую признательность П. И. Пронину за полезные обсуждения и замечания, а также считаю своим приятным долгом поблагодарить В. В. Асадова за финансовую поддержку исследований.

\section{Список литературы}

[1] N. Seiberg, E. Witten. Nucl. Phys. B. 1994. V. 426. P. 19.

[2] D. Finnell, P. Pouliot. Nucl. Phys. B. 1995. V. 453. P. 225.

[3] A. Yung. Nucl. Phys. B. 1997. V. 485. P. 38.

[4] N. Dorey, V. Khoze, M. Mattis. Multi-instanton calculus in $N=2$ supersymmetric gauge theory. hep-th/9603136.

[5] H. Nilles. Phys. Rep. 1984. V. 110. P. 1.

[6] I. Affleck, M. Dine, N. Seiberg. Nucl. Phys. B. 1984. V. 241. P. 493.

[7] N. Seiberg. Phys. Rev. D. 1994. V. 49. P. 6857.

[8] P. Pronin, K. Stepanyantz. Anomalies and nonperturbative results. hep-th/9803185.

[9] П. Пронин, К. Степаньянц. ТМФ. 1999. Т. 120. С. 82.

[10] G. Veneziano, S. Yankelowicz. Phys. Lett. B. 1982. V. 113. P. 231; T. Taylor, G. Veneziano, S. Yankelowicz. Nucl. Phys. B. 1983. V. 218. P. 493.

[11] K. Intriligator, R. Leith, N. Seiberg. Phys. Rev. D. 1994. V. 50. P. 1092.

[12] П. Пронин, К. Степаньяни. ТМФ. 1998. Т. 115. С. 402.

[13] G. Bonelli, M. Matone, M. Tonin. Phys. Rev. D. 1997. V. 55. P. 6466.

[14] P. Howe, P. West. Nucl. Phys. B. 1997. V. 486. P. 425.

[15] L. Alvares-Gaume, S. Hassan. Fortschr. Phys. 1997. V. 45. P. 159; hep-th/9701069.

Поступила в редакцию 5.I.1999 г., после доработки 21.V.1999 г. 